\section{The Roth Project - Music and Memory: A Community Agency Initiated Individualized Music Intervention for People with Dementia}

Emmali Buller, M.S. ${ }^{1}$, Phillip K. Martin, Ph.D. ${ }^{2}$, Anthony Stabler, Psy.D. ${ }^{3}$, Breana Tucker, MSW ${ }^{4}$, Jenna Smith, MPH, M.A. ${ }^{4}$, Linsey Norton, $\mathrm{MSW}^{5}$,

Ryan W. Schroeder, Psy.D. ${ }^{2}$

'Saint Francis Ministries, Salina, KS

${ }^{2}$ University of Kansas School of Medicine-Wichita, Department of Psychiatry and Behavioral Sciences, Wichita, KS

${ }^{3}$ McLean Hospital, Belmont, MA

${ }^{4}$ Alzheimer's Association of Central and Western Kansas Chapter, Wichita, KS

${ }^{5}$ Heart Centered Counseling, Fort Collins, CO

Received Feb. 11, 2019; Accepted for publication. June 24, 2019; Published online Nov. 25, 2019

\section{ABSTRACT}

Introduction. "The Roth Project - Music and Memory" is a musicbased intervention program, implemented by the Alzheimer's Association of Central and Western Kansas, which provides a nonpharmacological means for addressing neuropsychiatric symptoms in individuals with dementia.

Methods. Participants were individuals with dementia who were enrolled in The Roth Project - Music and Memory. Post-intervention surveys were distributed to caregivers of participants which assessed caregiver satisfaction with the program as well as caregiver perception of the impact of individualized music on mood and behavioral symptoms.

Results. Of returned surveys ( $\mathrm{n}=79), 99 \%$ of caregivers indicated they were satisfied or very satisfied with the program and $94 \%$ of caregivers perceived participants to like or very much like listening to the music. While a substantial number of participants required assistance with iPod use (95\%), the majority of participants were observed to listen to the music with stable or increased frequency over time. Personalized music was observed to improve mood in $78 \%$ of cases, with the most frequent benefits being improved overall happiness, decreased anxiety, increased positive emotional expression, and decreased depression.

Conclusions. The Roth Project - Music and Memory was well received by caregivers and was perceived to benefit mood of individuals with dementia. These results provided ongoing support for individualized music-based interventions and demonstrated that such interventions, when implemented by community agencies, can be well received by those who use them.

\section{Kans J Med 2019;12(4):136-140.}

\section{INTRODUCTION}

According to the World Health Organization, there are 50 million individuals suffering from dementia across the world, and approximately 20 million new cases are diagnosed each year. ${ }^{1}$ In addition to memory loss and other cognitive changes, the majority of

\section{KANSAS JOURNAL of MEDICINE}

individuals with dementia will experience neuropsychiatric symptoms including agitation, mood dysregulation, and thought disturbance., ${ }^{2,3}$ These symptoms contribute to a higher rate of hospitalization and caregiver burden. ${ }^{4}$ Pharmacological agents such as antipsychotics, anticonvulsants, and antidepressants are utilized commonly to treat neuropsychiatric symptoms among community-dwelling individuals with dementia; unfortunately, these medications can induce undesirable and potentially harmful side effects including cardiac problems, accelerated cognitive decline, drowsiness, and increased fall risk. ${ }^{5}$ The desire to reduce both the increased use of medical care and undesirable side effects of pharmacological agents has prompted researchers to explore a variety of nonpharmacological approaches aimed at treating neuropsychiatric symptoms among individuals with dementia. ${ }^{6}$

Music-based interventions are one type of nonpharmacological treatment approach which have received research support for reducing neuropsychiatric symptoms among individuals with dementia. ${ }^{7-10}$ Of music-based interventions, individualized music-based interventions may be a particularly useful method for reducing neuropsychiatric symptoms; these interventions are characterized by having the individual with dementia listen to familiar and personally preferred music from their past, often on an iPod or MP3 player., ${ }^{71,12}$ These interventions are thought to be beneficial due to helping individuals with dementia focus their attention on a stimulus (i.e., music). This, in turn, increases arousal regulation and stimulates remote memory by eliciting positive emotions that previously have been linked to the music, provides a known stimulus to override confusing stimuli in the immediate environment, promotes relaxation and reduces stress, and acts as a form of self-expression. ${ }^{13-16}$

Individualized music-based interventions can be convenient for community-dwelling individuals with dementia because these interventions can be personalized to the musical preferences of the individual, do not require services of a credentialed professional, and are accessible at any time of the day; thus, they are convenient, costeffective, and non-invasive. ${ }^{17}$ In an early study, investigators explored the benefits of implementing a personalized music intervention in the form of providing nostalgic music via a programmed jukebox to a small group of individuals with Alzheimer's disease at a Senior Care and Activities Center day program. ${ }^{18}$ The authors found that participants were able to access the music easily with minimal staff assistance, and that the easy-to-access format encouraged choice and some level of independence. Moreover, participants preferred the music listening activity over physical activities, arts and crafts, reading, and games. In another study, ${ }^{17}$ researchers utilized a previously published individualized music protoco ${ }^{19}$ in a group of 15 home-dwelling older adults with dementia and symptoms of agitation. The researchers found that agitation was decreased while participants were listening to music compared to times when they were not listening to music. 
KANSAS JOURNAL of MEDICINE

THE ROTH PROJECT

continued.

While the impact of individualized music-based interventions on mood and agitation has been researched previously, ${ }^{7-10}$ the authors of the current study are unaware of any study investigating whether such an intervention might be successful if initiated by a community organization. Instead, the majority of studies to date have been implemented primarily by independent researchers, with limited discussion of potential widespread dissemination and usage of music-based interventions. Thus, the current project demonstrated that an individualized music-based intervention can be well received by caregivers of individuals with dementia and perceived to reduce neuropsychiatric symptoms when initiated by a community-based organization.

\section{METHODS}

The "Roth Project - Music and Memory" (from this point forward referred to as the "Roth Project") was founded by a Wichita State University graduate student (author LN) in collaboration with the Alzheimer's Association of Central and Western Kansas and staff from a local memory care facility. This program was modeled after the original "Music and Memory" program founded in 2008 by Dan Cohen, ${ }^{20}$ but it was tailored specifically to clients of the Alzheimer's Association of Central and Western Kansas. Through this service, the Alzheimer's Association of Central and Western Kansas distributed iPods with personalized playlists to individuals with diagnosed or suspected dementia. Initial funding for the project came from several small grants and donations of music gift cards and used iPod shuffles and CDs. Families of participants in the program also were given the voluntary option of donating $\$ 60$ at enrollment to cover the initial cost of an iPod and music.

Playlists typically were chosen by the participant's informed caregiver during the enrollment interview and usually averaged from 15 - 30 songs (occasionally, an individual who was in the early stages of dementia would be able to choose his or her own playlist). Targeted songs were generally those listened to by the participants in their late teens or early twenties. If the caregivers did not know what kind of music to pick, they were asked questions about the participant's favorite type of music such as: what kind of concerts they went to, whether they enjoyed hymns, and if they had a special song from their wedding.

Agency staff or practicum students met with caregivers and utilized the Cohen-Mansfield Agitation Inventory (CMAI) along with a psychosocial evaluation to determine target agitation behaviors, and to provide instructions for when and how to use the iPod. It generally was recommended that participants use the iPod 20 - 30 minutes during an active time of day, as well as at the first sign of agitation. Once enrolled in the program, caregivers were provided follow-up phone calls at one month, three months, and six months to address management of behavioral issues, enhance use of the iPod, and to make adjustments, if needed, to the music playlist or listening schedule of the participants.
Approximately 2.5 years after the Roth Project was initiated, surveys were disseminated by the Alzheimer's Association of Central and Western Kansas as part of a quality improvement (QI) project. The intent of the surveys was to monitor outcomes and gauge benefit from the Roth Project to justify its continued utility as a local community support. Given this QI purpose, institutional review board oversight was not required or obtained.

Participants. Eligible participants were identified retrospectively and included all individuals who were enrolled in the Roth Project service from March 7, 2013 to November 23, $2015(\mathrm{n}=209)$. The individuals no longer participating in the Roth Project at the time of survey dissemination were excluded and their care providers were not sent a survey. This included 19 individuals who had withdrawn from the Roth Project due to no longer wanting to participate, not utilizing the iPod, or who had lost the iPod. Of the remaining 190 eligible individuals, surveys were sent to caregivers of the participants thought to be living, and 79 surveys were returned. Thus, the response rate was at least $42 \%$, although likely higher given that an unknown proportion of the participants passed away prior to the study survey, precluding their caregivers from receiving a survey. The surveys were anonymous and no identifying information was collected. Survey participation was voluntary and completion did not influence continued enrollment in the Roth Project.

Specific descriptive/demographic information was not collected for participants upon enrollment in the Roth Project, nor was this information collected in the subsequent survey. This was to keep the survey as brief as possible given that it was disseminated for QI purposes. In general, those who received the music-based intervention were community-dwelling individuals with diagnosed or suspected dementia who ranged in age from late-fifties to mid-nineties. According to the Alzheimer's Association of Central and Western Kansas staff who enrolled participants in the Roth Project, the majority of participants were Caucasian, native English-speaking individuals. Participants resided in the South-Central region of Kansas. Most individuals were referred to the Roth Project program by caregivers but, occasionally, those who were in the earlier stages of dementia enrolled in the program themselves. Assent was obtained from participants upon enrollment.

Survey. A survey (Appendix A) containing six questions was distributed to caregivers of individuals with diagnosed or suspected dementia who were living and remained enrolled in the program. Five out of six items on the survey were rated on an ordinal scale. Questions regarded caregiver satisfaction with the program, participant reaction to the program, frequency of iPod usage, effects of music on participant mood, and percentage of time that the participant required assistance from the caregiver in using the iPod. The last question of the survey asked caregivers to select from various potential benefits which improvements in mood, behavior, or functioning were observed due to listening to the music. Several of the potential improvements were improved attentiveness, decreased depression and irritability, and increased cooperation, positive emotional expression, positive interactions with others, and overall happiness. 


\section{RESULTS}

Following data collection, survey information was entered into IBM SPSS Statistics 23 and frequency data were analyzed. Of the returned surveys, 78 of 79 caregivers were satisfied or very satisfied with the program. Additionally, the majority of caregivers (94\%) believed that the participant liked (50\%) or very much liked (44\%) listening to the personalized music playlists. Approximately one-half (49\%) of program participants were observed to listen to their iPods with stable frequency over time, $11 \%$ of participants were observed to listen to their iPods with increasing frequency, and $39 \%$ were observed to listen with less frequency. The majority of participants (70\%) required assistance with their iPods at each use, $25 \%$ required help in some but not all cases, and $5 \%$ were observed to use their iPod consistently without assistance.

For most participants (78\%), personalized music was observed by caregivers to improve overall mood (Figure 1). No effect on mood was observed in $18 \%$, and a worsening of mood was observed in $4 \%$ of individuals. Among the cases where improvement in mood was observed, caregivers most frequently endorsed benefits of overall happiness $(71 \%)$, decreased anxiety (68\%), increased positive emotional expression (55\%), decreased depression (36\%), increased positive interactions (34\%), and increased energy and arousal (34\%).

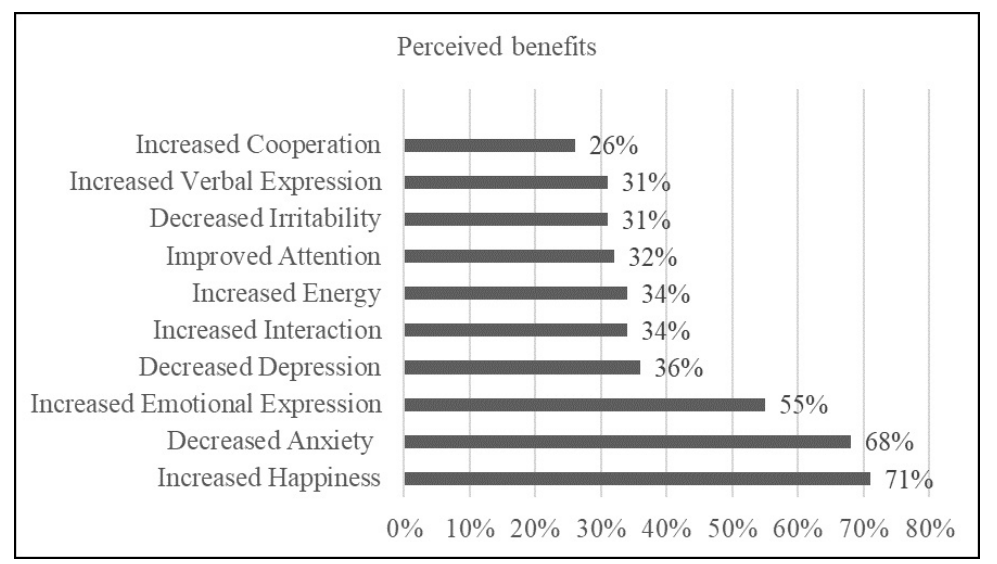

Figure 1. Perceived benefits of the Roth Project.

\section{DISCUSSION}

The current project sought to examine whether an individualized music-based intervention, when initiated by a community-based organization, would be well received and viewed as helpful in reducing neuropsychiatric symptoms by caregivers of individuals with dementia. Overall, the program was well-received by caregivers and well-tolerated by participants. Nearly all surveyed caregivers indicated that they observed improvements in the neuropsychiatric status of participants, with the most commonly reported improvement being increased overall happiness.

Due to the positive caregiver response and impact of music on participant mood, it is likely that similar results can be achieved in other communities. These are promising findings given that individualized music-based interventions, such as the Roth Project, can be relatively inexpensive to both community organizations and participating individuals. Startup costs for the Roth Project were minimal and derived from small grants and donations. The Roth Project has continued to subsist on family donations and memorials and is providing
KANSAS JOURNAL of MEDICINE

THE ROTH PROJECT

continued.

individualized music programs successfully to individuals with dementia located in central Kansas. Labor costs for community programs providing individualized music also can be minimal. Roth Project staffing has been supported largely by university practicum students, and programs such as this can offer an enjoyable opportunity for community service to students and volunteers alike.

In developing programs similar to the Roth Project, interested organizations should keep in mind several considerations. First, our data indicated that the majority of individuals with dementia will be unable to utilize the iPod without help. As a result, it likely will be beneficial to show each caregiver how to use the iPod and provide a list of specific situations where the music playlist might be calming or engaging for the person with dementia. The caregiver should be prompted to offer the iPod in these situations. Second, the iPods easily can be misplaced. Thus, it may be helpful to provide a small bag, carrying case, or another tool that encourages the participant or caregiver to carry the device with them or keep it within close reach. Similarly, if the participant lives in a care facility, it could be useful to keep the iPod and headphones in a discreet case attached to the person's wheelchair, bedframe, walker, or door where it will be out of the way, but potentially serve as a reminder for facility caregivers to utilize the music intervention. Third, it is recommended that future programs consider offering alternative ways of playing music playlists in addition to utilizing headphones and iPod shuffles. Some individuals were not able to utilize the music intervention regularly due to not being able to leave the headphones alone, continually trying to take them off, or pulling on the cord. Alternatively, Bluetooth enabled cordless headphones could be considered.

Limitations. Several limitations regarding study methodology warrant discussion. The rate of survey response could not be determined, as the exact number of participants receiving a survey was unknown. Given that it is estimated that no more than 190 participants were eligible to receive the survey and that 79 surveys were returned, it can be assumed that the response rate was at least $42 \%$. This is likely an underestimate of the true response rate given that some proportion of potentially eligible participants were likely excluded due to passing away.

The impact of attrition should be considered when examining the current study findings. A minority of the participants $(\mathrm{n}=16)$ left the program because they were no longer interested in using their iPods; had survey data been collected for such participants, it is likely that the proportion of respondents providing positive feedback would have been somewhat less. That being said, the majority of enrolled individuals continued to utilize the program with data suggesting that most of these individuals were observed to experience benefit. Also of note, approximately $41 \%$ of initial enrollees passed away at some point following enrollment, excluding their caregivers from completing a survey. This group likely represented individuals who were more advanced in their dementia, whose health status was complicated by 
KANSAS JOURNAL of MEDICINE THE ROTH PROJECT

continued.

\section{REFERENCES}

${ }^{1}$ World Health Organization. Dementia 2017. Available at: http://www.who. int/news-room/fact-sheets/detail/dementia. Accessed September 9, 2018.

2 Cheong CY, Tan JA, Foong YL, et al. Creative music therapy in an acute care setting for older patients with delirium and dementia. Dement Geriatr Cogn Dis Extra 2016; 6(2):268-275. PMID: 27489560.

${ }^{3}$ Gruber-Baldini AL, Boustani M, Sloane PD, Zimmerman S. Behavioral symptoms in residential care/assisted living facilities: Prevalence, risk factors, and medication management. J Am Geriatr Soc 2004; 52(10):1610-1617. PMID: 15450035.

${ }^{4}$ Sourial R, McCusker J, Cole M, Abrahamowicz M. Agitation in demented patients in an acute care hospital: Prevalence, disruptiveness, and staff burden. Int Psychogeriatr 2001; 13(2):183-197. PMID: 11495393.

5 Buchalter EN, Lantz MS. Treatment of impulsivity and aggression in a patient with vascular dementia. Geriatrics 2001; 56(2):53-54. PMID 11219025.

${ }^{6}$ Olazarán J, Reisberg B, Clare L, et al. Nonpharmacological therapies in Alzheimer's disease: A systematic review of efficacy. Dement Geriatr Cogn Disord 2010; 30(2):161-178. PMID: 20838046.

7 Schroeder RW, Martin PK, Marsh C, et al. An individualized music-based intervention for acute neuropsychiatric symptoms in hospitalized older adults with cognitive impairment: A prospective, controlled, nonrandomized trial. Gerontol Geriatr Med 2018; 4:2333721418783121. PMID: 29977982.

${ }^{8}$ Sung HC, Chang AM, Abbey J. The effects of preferred music on agitation of older people with dementia in Taiwan. Int J Geriatr Psychiatry 2006; 21(10):999-1000. PMID: 16998779.

9 Svansdottir HB, Snaedal J. Music therapy in moderate and severe dementia of Alzheimer's type: A case-control study. Int Psychogeriatr 2006; 18(4):613621. PMID: 16618375.

10 Vasionytė I, Madison G. Musical intervention for patients with dementia: A meta-analysis. J Clin Nurs 2013; 22(9-10):1023-1016. PMID: 23574287.

${ }^{11}$ Garland K, Beer E, Eppingstall B, O'Connor DW. A comparison of two treatments of agitated behavior in nursing home residents with dementia: Stimulated family presence and preferred music. Am J Geriatr Psychiatry 2007; 15(6):514-521. PMID: 17293386.

${ }^{12}$ Sung HC, Chang AM, Lee WL. A preferred music listening intervention to reduce anxiety in older adults with dementia in nursing homes. J Clin Nurs 2010; 19 (7-8):1056-1064. PMID: 20492050.

${ }^{13}$ Brotons M, Koger SM. The impact of music therapy on language functioning in dementia. J Music Ther 2000; 37(3):183-195. PMID: 10990596.

${ }^{14}$ Gerdner LA. Individualized music for dementia: Evolution and application of evidence-based protocol. World J Psychiatry 2012; 2(2):26-32. PMID: 24175165.

${ }^{15}$ McDermott O, Crellin N, Ridder HM, Orrell M. Music therapy in dementia: A narrative synthesis systematic review. Int J Geriatr Psychiatry 2013; 28(8):781-794. PMID: 23080214.

${ }^{16}$ Vink AC, Birks JS, Bruinsma MS, Scholten RJ. Music therapy for people with dementia. Cochrane Database Syst Rev 2004; (3):CD003477. PMID: 15266489.

${ }_{17}$ Park H, Pringle-Specht JK. Effect of individualized music on agitation in individuals with dementia who live at home. J Gerontol Nurs 2009; 35(8):4755. PMID: 19681563.

18 Olsen RV, Hutchings BL, Ehrenkrantz E. "Media memory lane” interventions in an Alzheimer's day care center. Am J Alzheimers Dis 2000; 15(3):163-175.

19 Gerdner LA. Use of individualized music by trained staff and family: translating research into practice. J Gerontol Nurs 2005; 31(6):22-30. PMID: 16138527.

${ }^{20}$ Music \& memory. Our Mission and Vision 2018. Available at: https:// musicandmemory.org/about/mission-and-vision/. Accessed October 12, 2018.

${ }^{21}$ Kelley K, Clark B, Brouwn V, Sitzia J. Good practice in the conduct and reporting of survey research. Int J Qual Health Care 2003; 15(3):261-266. PMID: 12803354.

Keywords: music therapy, dementia, memory disorders, community-based participatory research 


\section{Roth Project Survey}

1. Overall, how satisfied are you with the iPod program? Very Satisfied Satisfied Dissatisfied Very Dissatisfied

2. How did the resident respond to listening to the iPod?

Very Much Liked Disliked Very Much Disliked

3. Over the course of the month (Please choose one):

a. The resident listened to the iPod the same frequency over time

b. The resident listened to the iPod less often over time

c. The resident listened to the iPod more often over time

4. What effect did listening to the iPod have on mood?

Very Positive Positive No Response Negative Very Negative

5. About what percent of the time does the resident require help with the iPod when using it?

$100 \% \quad 75 \% \quad 50 \% \quad 25 \% \quad 0 \%$

6. In which of these areas did listening to iPod result in improvement?
a. Depression
b. Cooperation
c. Anxiety
d. Attentiveness
e. Pain
f. Positive interactions with others
g. Irritability
h. Overall happiness
i. Thoughts related to suicide or death
j. Verbal expression
k. Positive emotional expression
l. Energy/arousal 